\title{
Eddy length scales in the North Atlantic Ocean
}

\author{
Carsten Eden ${ }^{1}$
}

Received 28 August 2006; revised 8 December 2006; accepted 20 December 2006; published 7 June 2007.

[1] Eddy length scales are calculated from satellite altimeter products and in an eddy-resolving model of the North Atlantic Ocean. Four different measures for eddy length scales are derived from kinetic energy densities in wave number space and spatial decorrelation scales. Observational estimates and model simulation agree well in all these measures near the surface. As found in previous studies, all length scales are, in general, decreasing with latitude. They are isotropic and proportional to the local first baroclinic Rossby radius $\left(L_{\mathrm{r}}\right)$ north of about $30^{\circ} \mathrm{N}$, while south of $30^{\circ} \mathrm{N}$ ( or for $L_{\mathrm{r}}>30 \mathrm{~km}$ ), zonal length scales tend to be larger than meridional ones, and (scalar) length scales show no clear relation to $L_{\mathrm{r}}$ anymore. Instead, they appear to be related to the local Rhines scale. In agreement with a recent theoretical prediction by Theiss [2004], the observed and simulated pattern of eddy length scales appears to be indicative of two different dynamical regimes in the North Atlantic: anisotropic turbulence in the subtropics and isotropic turbulence in the subpolar North Atlantic. Both regions can be roughly characterized by the ration between $L_{\mathrm{r}}$ and the Rhines scales $\left(L_{\mathrm{R}}\right)$, with $L_{\mathrm{R}}>L_{\mathrm{r}}$ in the isotropic region and $L_{\mathrm{R}}<L_{\mathrm{r}}$ in the anisotropic region. The critical latitude that separates both regions, i.e., where $L_{\mathrm{R}}=L_{\mathrm{r}}$, is about $30^{\circ} \mathrm{N}$.

Citation: Eden, C. (2007), Eddy length scales in the North Atlantic Ocean, J. Geophys. Res., 112, C06004, doi:10.1029/2006JC003901.

\section{Introduction}

[2] Values for lateral turbulent diffusivities used today in ocean general circulation models, such as the isopycnal thickness diffusivity by Gent and McWilliams [1990], are still rather uncertain [Bryan et al., 1999; Eden et al., 2006]. It is obvious, however, that the specific choice of a value for such diffusivities affects the simulations of non-eddyresolving ocean models, which are typically used for climate research, in particular, for projections of the evolution of the climate for the next 100 years [Houghton et al., 2001]. The magnitude of the turbulent diffusivities is often inferred from the product of a characteristic velocity scale and a characteristic length scale of the largest eddies in a turbulent flow field [Green, 1970; Stone, 1972; Larichev and Held, 1995; Thompson and Young, 2006], a concept going back to Prandtl [1925]. A characteristic near-surface velocity scale for mesoscale eddies in the ocean can be obtained from observational estimates of eddy kinetic energy, given, for example, by satellite altimeter data [Stammer, 1997]. Since recent eddy-resolving ocean models show remarkably good agreement with observations in the simulation of mesoscale variability [Smith et al., 2000; Eden and Böning, 2002], the characteristic velocity scale at depth can be readily inferred from such simulations.

\footnotetext{
${ }^{\mathrm{I}}$ FB I Ocean Circulation and Climate Dynamics, IFM-GEOMAR, Kiel, Germany.

Copyright 2007 by the American Geophysical Union. 0148-0227/07/2006JC003901\$09.00
}

[3] On the other hand, eddy length scales in the ocean are less well known. Le Traon et al. [1990] and Stammer and Böning [1992] noted large geographical variations of characteristic eddy length scales inferred from spatial autocorrelation function of satellite observations of sea surface height variability. In both studies, the latitudinal variations of the length scale were thought to be indicative of a linear relation with the first baroclinic Rossby radius, although it was also noted by Le Traon [1993] that while the Rossby radius varies by a factor of 4 , the length scales vary only by a factor of 2. Restricted to the northern North Atlantic, however, Krauss et al. [1990], using surface drifter data and infrared satellite images, reported a linear relation between Rossby radius and eddy length scale. Ocean model simulations tend to show similar spectral characteristics as the satellite observations, although low resolution in midlatitudes due to computational restrictions hampered the model analysis for a long time [Stammer and Böning, 1992]. However, recent high-resolution eddy-resolving models have shown remarkably close correspondence to the observations with respect to mesoscale variability [Beckmann et al., 1994; Smith et al., 2000].

[4] In this study, the question regarding a possible relation between the eddy length scale and the Rossby radius is revisited, based on recent satellite data products and a stateof-the-art eddy-resolving model of the North Atlantic Ocean. A clear linear relation is found only for Rossby radii smaller than $30 \mathrm{~km}$ or north of $30^{\circ} \mathrm{N}$ in the North Atlantic. Section 2 presents details of the model simulation, the observational data, and the methods used to explore the spectral properties of the turbulent oceanic flow, section 3 presents the results of the analysis, while in section 4 , the 
conclusions of the present study are summarized and discussed.

\section{Methods and Data}

[5] To estimate the spectral properties of the turbulent flow, pressure $p$ is used in this study which acts in the quasigeostrophic approximation as a stream function for the geostrophic velocity, $\boldsymbol{u}$, i.e., as $f \boldsymbol{e}_{3} \times \boldsymbol{u}=-\nabla_{\mathrm{h}} p$ where $f$ denotes the (fixed) Coriolis parameter. Furthermore, the surface pressure $p_{0}$ is related to the sea surface height $\zeta$ by $p_{0}=g \rho_{0} \zeta$, for which reliable measurements are given by satellite altimeter data. The Fourier transform of $p$ is given by

$$
P(\boldsymbol{k})=\int_{-\infty}^{\infty} p \mathrm{e}^{i \boldsymbol{k} \cdot \boldsymbol{x}} \mathrm{d} \boldsymbol{x}
$$

where $\boldsymbol{k}$ denotes the horizontal wave number vector and $\boldsymbol{x}$ denotes the horizontal position. For fixed latitude, the horizontal derivatives of $p$ are proportional to the geostrophic velocity $\boldsymbol{u}(\boldsymbol{x})$. The Fourier transform of $\boldsymbol{u}(\boldsymbol{x})$ is given by

$$
f \boldsymbol{e}_{3} \times \boldsymbol{U}(\boldsymbol{k})=f \boldsymbol{e}_{3} \times \int_{-\infty}^{\infty} \boldsymbol{u} \mathrm{e}^{i \boldsymbol{k} \cdot \boldsymbol{x}} \mathrm{d} \boldsymbol{x}=-i \boldsymbol{k} P
$$

The horizontal kinetic energy is given by $\frac{u^{2}}{2}$ and its Fourier transform $E(\boldsymbol{k})$ is given by

$$
2 E(\boldsymbol{k})=|U(\boldsymbol{k})|^{2}=|f|^{-2} k^{2} P^{2}
$$

where $k=|\boldsymbol{k}|$. The scalar energy density in wave number space, $E(k)$, is given by

$$
E(k)=\int_{0}^{2 \pi} E(k, \phi) \mathrm{d} \phi
$$

where $\tan \phi=\frac{k_{y}}{k_{x}}$. The (scalar) spatial autocovariance function $R(\tau)$ of $\boldsymbol{u}$ at lag $\tau$ is given by

$$
R(\tau)=\int_{-\infty}^{\infty} E(k) \mathrm{e}^{-i k \tau} \mathrm{d} k
$$

where $\tau$ denotes a horizontal lag. Note that at depth $z$, the full pressure $p=p_{0}+\int_{z}^{0} g \rho \mathrm{d} z$ replaces the surface pressure $p_{0}$ as the stream function for the geostrophic velocity $\boldsymbol{u}(\boldsymbol{x}, z)$ such that $P, U, E$, and $R$ become functions of depth.

[6] The Fourier transforms are estimated on a discrete grid for both an eddy-resolving model and satellite altimeter data (while the latter is used for the case of the surface pressure only). The horizontal resolution $\left(1 / 12^{\circ}\right)$ of the model is about $10 \mathrm{~km}$ at the equator decreasing to about $5 \mathrm{~km}$ in high latitudes. The model domain extends from $20^{\circ} \mathrm{S}$ to $70^{\circ} \mathrm{N}$ with open boundaries [Stevens, 1990] at the northern and southern boundaries and with a buoyancy restoring zone in the eastern Mediterranean Sea. There are 45 vertical geopotential levels with increasing thickness with depth, ranging from $10 \mathrm{~m}$ at the surface to $250 \mathrm{~m}$ near the maximal depth of $5500 \mathrm{~m}$. Surface boundary forcing is given by monthly mean wind stress, a Haney-type heat flux condition as given by
Barnier et al. [1995], and a restoring condition for sea surface salinity. The model is based on a rewritten version (FLAME; the numerical code together with all configurations used in this study can be accessed at http://www.ifm-geomar.de/ $\sim$ spflame) of MOM2 [Pacanowski, 1995] and is identical to the one used, e.g., in the work of Eden [2006a]. The model is integrated for a 10-year spin-up period; the results shown here are taken from a subsequent integration of 5 years. The satellite altimeter data set comes from the JASON and TOPEX/POSEIDON missions. The altimeter products have been produced by SSALTO/DUACS and are distributed by AVISO on a regular $1 / 4^{\circ} \times 1 / 4^{\circ}$ grid. They have been downloaded from the AVISO web page for the years 2003-2004 for JASON and 1993-2002 for TOPEXPOSEIDON.

[7] The data are interpolated from each grid on different subregions with meridional extent of $\phi_{0} \leq \phi \leq \phi_{0}+12^{\circ}$ and zonal extent of $\lambda_{0} \leq \lambda \leq \lambda_{0}+12^{\circ}\left(\cos \phi_{m}\right)^{-1}$, where $\phi$ denotes latitude, $\lambda$ denotes longitude, $\phi_{m}=\phi_{0}+6^{\circ}$, and $\phi_{0}$ and $\lambda_{0}$ are the coordinates of the southwestern most point. Each subregion is discretized with grid spacing of $\Delta \phi=$ $\Delta \lambda\left(\cos \phi_{m}\right)^{-1}$. Note that the cosine factor ensures that the spatial extent of the domain is approximately quadratic and the grid spacing is approximately isotropic. A discrete twodimensional Fourier transform (FFT) in space is applied for the pressure $p$ to obtain its Fourier transform $P$ in each subregion and for each time step. Prior to the FFT, the spatial mean of $p$ in the subregion and the temporal mean of $p$ is removed, but no window or envelope was applied to the data. The impact of the latter choice is discussed below. Resulting squared amplitudes of $P$ are averaged over time to apply statistical significance to the spectral estimates. For the model, data are used in a 3-day interval for 5 years; the satellite data are given in a 7-day interval for 2 (9) years in case of JASON (TOPEX/POSEIDON). The statistical significance of the estimation was assessed by using a subset of years for the analysis for the individual data sets (JASON, TOPEX/POSEIDON, or model), but the results remain almost identical.

[8] A note on the quality of the satellite data product is mandatory. It is well known that the temporal and spatial along-track and cross-track resolutions of both satellite altimeter are rather different. Here the gridded data product as provided by SSALTO/DUACS and AVISO is used, which has certainly less resolution than using the raw alongor cross-track data, because of smoothing effects during the interpolation procedure. A major concern is that the effective resolution of the gridded satellite data is close to the estimated eddy length scales. Note also that both satellite data and model data are interpolated on a common (isotropic) grid. It is clear that, in particular, in the subpolar North Atlantic, the results can be influenced by interpolation effects. The impact of this error will be assessed and discussed below by changing (increasing) the grid spacing $\Delta \lambda, \Delta \phi$ of each subregion.

\section{Results}

\subsection{Definition of Eddy Length Scales}

[9] Figure 1a shows the normalized spectral density $E(\boldsymbol{k})$ and Figure $1 \mathrm{~b}$ shows the normalized scalar spectral density $E(k)$, estimated from satellite altimeter data for a represen- 
a)

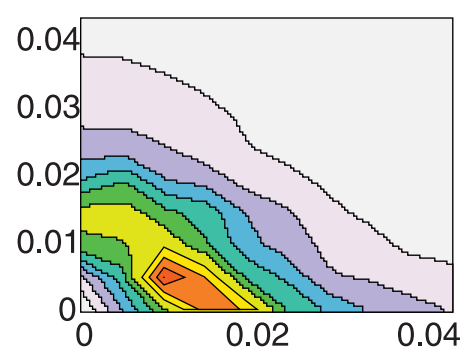

b)

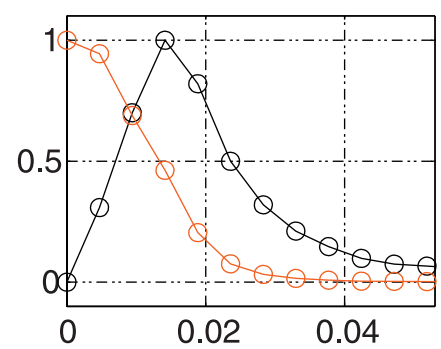

c)

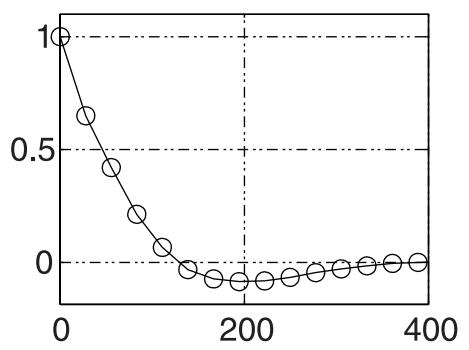

Figure 1. (a) Normalized spectral kinetic energy density $E(\boldsymbol{k})$ in a $12 \cos \phi_{m} \times 12^{\circ}$ subregion with southern/westernmost point at $\psi_{0}=25^{\circ} \mathrm{N}$ and $\lambda_{0}=52^{\circ} \mathrm{W}$ in JASON altimeter data as a function of wave number $k_{x}$ and $k_{y}$. Wave numbers are in $\mathrm{km}^{-1}$, contour spacing is 0.1 . (b) Normalized scalar spectral kinetic energy density $E(k)$ (black) and corresponding pressure spectrum $P^{2}(k)$ (red) in the same subregion. Scalar wave number $k$ is in $\mathrm{km}^{-1}$; the circles denote actual data points. (c) Normalized autocovariance function $R(\tau)$ in the same subregion. Lags $\tau$ are in $\mathrm{km}$.

tative subregion of the subtropical North Atlantic. There is a peak at a scalar wave number of about $0.015 \mathrm{~km}^{-1}$, corresponding to a wavelength of about $400 \mathrm{~km}$. Similar peaks can be seen in almost any other region of the North Atlantic, as shown in Figure 2. The wavelength at which $E(k)$ peaks varies, however, regionally. Clearly it can be assumed that this geographical variation of the maximum of $E(k)$ is related to a corresponding variation of the dynamics producing this spectral peak, i.e., to a variation in the characteristic length scale of the turbulent motion.

[10] There are several means to define a characteristic length scale in a spectral estimate. Most obvious from Figure $1 \mathrm{~b}$, one would use the scalar wave number, say $k_{\max }$, of the peak of $E(k)$ for the definition of a characteristic inverse length scale and $L_{\max }=2 \pi / k_{\max }$ as the corresponding length scale. On the other hand, it is also intuitive to look at the autocovariance function $R$, shown for example in Figure 1c. There is a rapid decay of covariance with increasing lags, with a typical first zero-crossing at about $130 \mathrm{~km}$. This first zero-crossing, say $L_{0}$, is also often used as a characteristic length scale [Stammer, 1997]. Here we

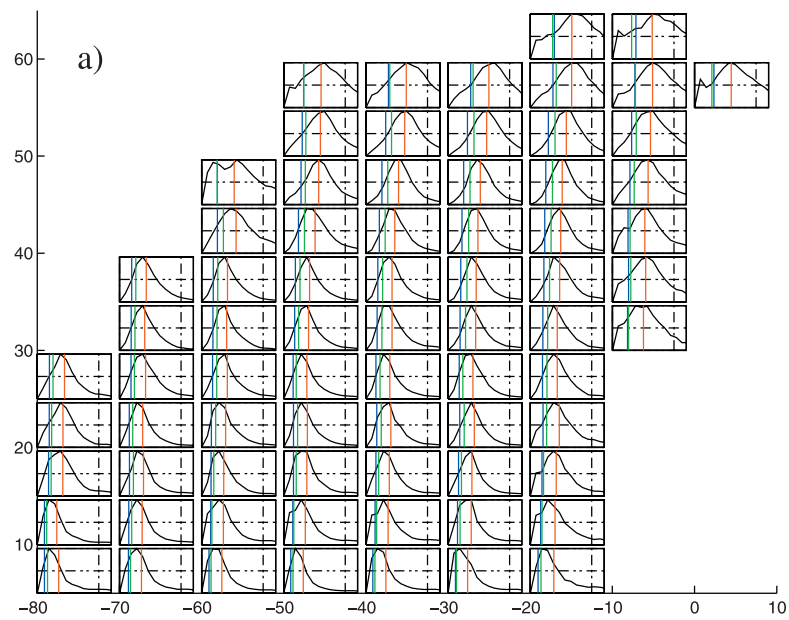

see that both heuristic measures are rather different in magnitude.

[11] To overcome the ambiguity of the more heuristic length scales, $L_{\max }$ and $L_{0}$, in classical homogeneous turbulence theory, the relevant length scale for the large, energy-containing eddies is often defined by the integral length scale

$$
L_{1}=R(0)^{-1} \int_{0}^{\infty} R(\tau) \mathrm{d} \tau
$$

[Batchelor, 1969]. Since $R$ is the Fourier transform of $E$, the integral length scale can also be expressed using the spectral energy density $E(k)$ as

$$
L_{1} \sim \frac{\int k^{-1} E(k) \mathrm{d} k}{\int E(k) \mathrm{d} k}=\frac{\int k P^{2}(k) \mathrm{d} k}{\int k^{2} P^{2}(k) \mathrm{d} k}
$$

[Batchelor, 1969]. Note that $L_{1}$ is akin to a "mean" wavelength in the energy spectrum. However, it is shown below that estimates of $L_{1}$ for the application of the present

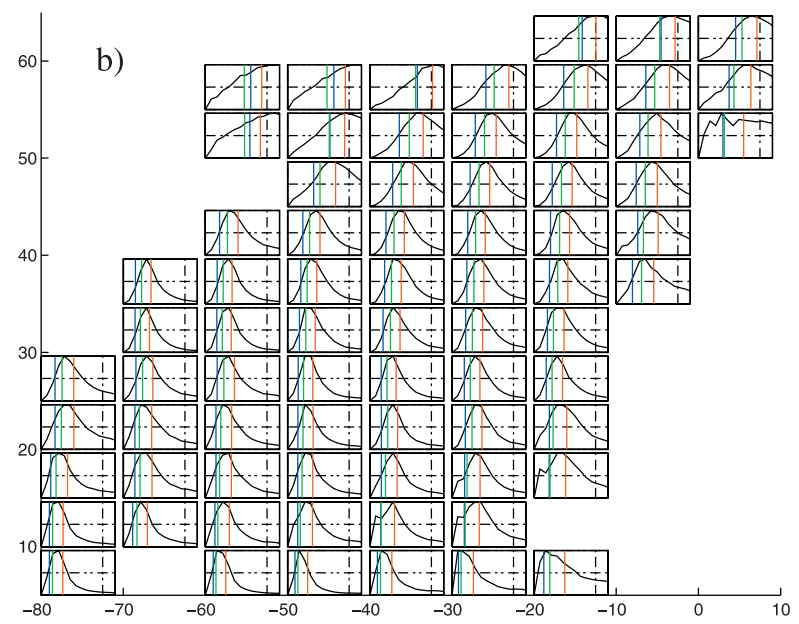

Figure 2. (a) Normalized wave number spectra $E(k)$ from JASON from 0 to $0.06 \mathrm{~km}^{-1}$ (black solid line) estimated in $12^{\circ} / \cos \phi \times 12^{\circ}$ subregions with $1 / 4^{\circ}$ isotropic grid spacing. The subregions are located as a subplot at its approximate geographic location. Also shown are $L_{1}^{-1}$ (red) and $L_{0}^{-1}$ (blue) and $k_{*}$ (green) as vertical lines. (b) Same as Figure 2 a but for the model. 
a)

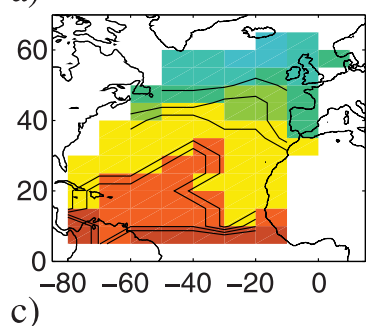

c)

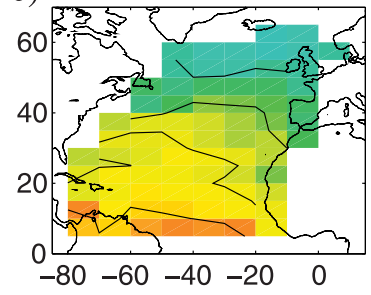

b)

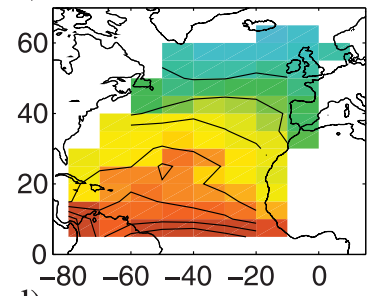

d)

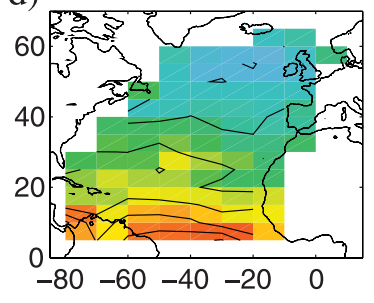

e)

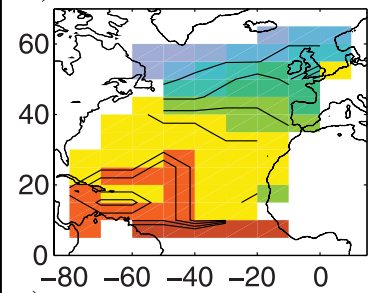

g)

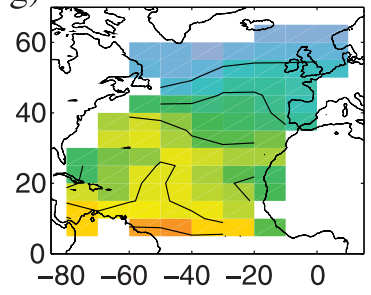

f)

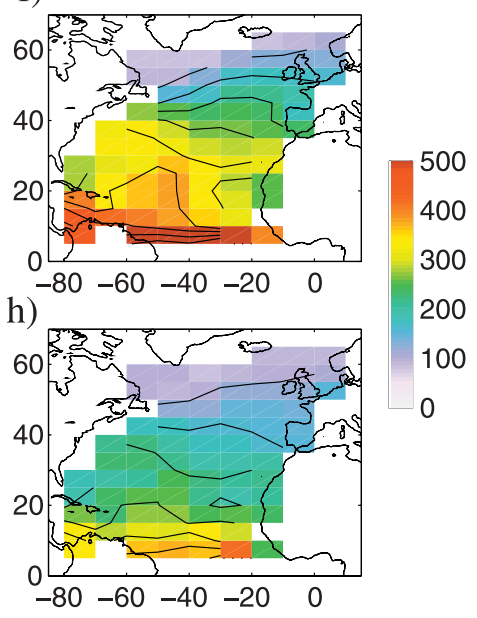

Figure 3. The length scales $L_{\max }(\mathrm{a}, \mathrm{e}), \pi L_{0}(\mathrm{~b}, \mathrm{f}), 2 \pi L_{1}(\mathrm{c}, \mathrm{g})$, and $0.5 L_{*}(\mathrm{~d}, \mathrm{~h})$ for JASON (a-d) and the model simulation $(\mathrm{e}-\mathrm{h})$ in $\mathrm{km}$. Contour spacing is $50 \mathrm{~km}$. Only subregions with more than $50 \%$ wet grid points are shown.

study depend heavily on the grid spacing, while the (inverse) length scale given by

$$
k_{*}=\frac{\int k P^{2}(k) \mathrm{d} k}{\int P^{2}(k) \mathrm{d} k}, L_{*}=2 \pi / k_{*}
$$

depends much less on the grid spacing. This is because the integral $\int k^{2} P^{2}(k) \mathrm{d} k$ relies much stronger on higher wave numbers than the integral $\int P^{2}(k) \mathrm{d} k$. This can also be seen in Figure $1 \mathrm{~b}$, in which $P(k)$ converges much more rapidly toward zero for high wave numbers than $E(k)=k^{2} P^{2}(k)$. Generalizing the concept for nonisotropic flow, it is also possible to define the following (inverse) vector length scale

$$
\begin{aligned}
\left(k_{*}^{(x)}, k_{*}^{(y)}\right) & =\boldsymbol{k}_{*}=\frac{\int \boldsymbol{k} P^{2}(k) \mathrm{d} k}{\int P^{2}(k) \mathrm{d} k} \\
\left(L_{*}^{(x)}, L_{*}^{(y)}\right) & =\boldsymbol{L}_{*}=\left(2 \pi / k_{*}^{(x)}, 2 \pi / k_{*}^{(y)}\right)
\end{aligned}
$$

In this study, the heuristic length scales $L_{\max }$ and $L_{0}$ will be shown for the satellite observations and model simulations, the integral length scales $L_{1}$ and $L_{*}$ will be discussed, and the vector length scale $\boldsymbol{L}_{*}$ will be used to detect a possible anisotropy in the turbulent flow.

\subsection{Eddy Length Scales in Observations and Model Simulation}

[12] Figure 2a shows the normalized energy spectra estimated in several subregions of the North Atlantic from JASON altimeter data. Also shown are the (inverse) length scales $L_{0}^{-1}, L_{1}^{-1}$, and $k_{*}$. Note that the corresponding estimate from TOPEX/POSEIDON is very similar to Figure $2 \mathrm{a}$ and is therefore not shown. It appears that 2 years of data from JASON give sufficient significance in the mean spectral estimates for the purpose of this study, such that in the remainder of the text, only the JASON satellite data will be discussed.

[13] In each subregion of the North Atlantic, there is a peak in $E(k)$ at a wave number $k_{\max }$ corresponding roughly to the integral length scale $L_{1}^{-1}$ (but note the missing factor $2 \pi)$. There is a clear increase in $k_{\max }$ with latitude. The first zero-crossing in the autocovariance function $R$, given by the length scale $L_{0}$, is roughly twice the integral length scale $L_{1}$ in each case. This is because the form of $R$ is in each subregion very similar to the one shown in Figure 1c, i.e., an almost linear decrease of $R$ toward the first zero-crossing at $L_{0}$ and small values of $R$ fluctuating around zero for larger lags than $L_{0}$. The scale $L_{0}^{-1}$ corresponds in Figure 2a roughly to the wave number $k_{*}$. As before for $k_{\max }$ and $L_{1}^{-1}$, there is also an increase of $L_{0}^{-1}$ and $k_{*}$ with latitude.

[14] Figure $2 b$ shows the normalized energy spectra for the model simulation. Note that analogous to the satellite data, the model data are interpolated on an isotropic $1 / 4^{\circ}$ grid prior to the analysis. The estimated form of the energy spectra and the corresponding length scales and their lateral variations are remarkably similar to the observations. However, in the subpolar North Atlantic, $k_{\max }$ and $L_{0}^{-1}$ tend to be larger compared to the observations. This is most likely because of too much smoothing in the observations involved during preparation of the gridded data product from the raw satellite tracks. Another reason might be insufficient horizontal resolution of the model in the northern subpolar gyre (compared to the local Rossby radius).

[15] Figure 3 shows all estimated length scales on a geographical plot in the North Atlantic to visualize the lateral dependencies of the length scales both for the satellite observations and the model simulation. Note that $L_{0}, L_{1}$, and $L_{*}$ are scaled by a factor $\pi, 2 \pi$, and 0.5 respectively, such that all length scales can be shown on the same color range. As before in Figure 2, it is evident that model and observation agree well in all measures. All (scaled) length scales vary similarly; that is, they are smaller than or equal to $100 \mathrm{~km}$ north of the subpolar front, increasing toward the equators to $500 \mathrm{~km}$. As seen before, in the subpolar North Atlantic, eddy length scales are smaller in the model (less than $100 \mathrm{~km}$ ) compared to the observations (larger than $100 \mathrm{~km}$ ), most likely because of the above-mentioned bias in the observations. 
a)

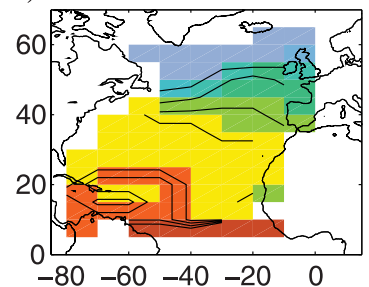

c)

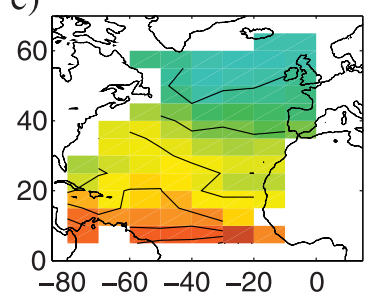

b)

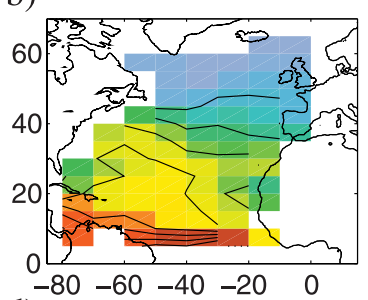

d)

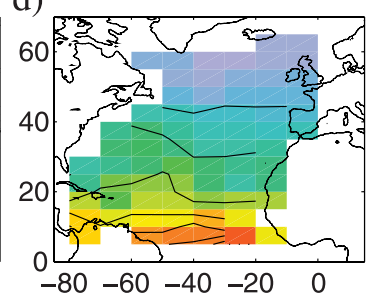

e)

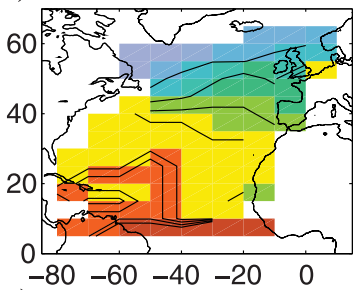

g)

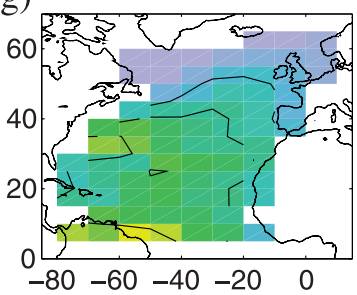

f)

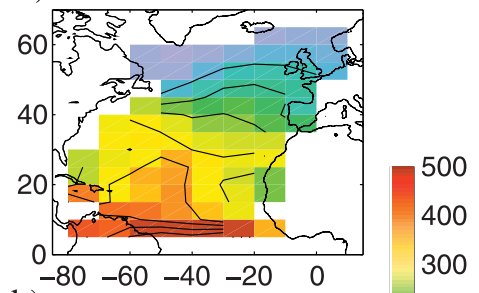

h)

$-80-60-40-20 \quad 0 \quad 300$

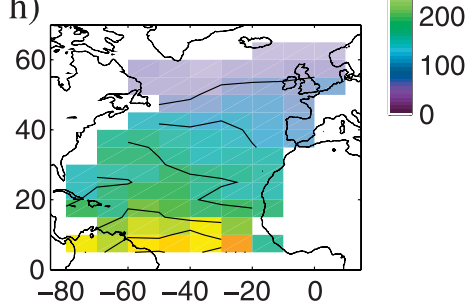

Figure 4. Length scales $L_{\max }(\mathrm{a}, \mathrm{e}), \pi L_{0}(\mathrm{~b}, \mathrm{f}), 2 \pi L_{1}(\mathrm{c}, \mathrm{g})$, and $0.5 L_{*}(\mathrm{~d}, \mathrm{~h})$ estimated from the model results interpolated on a $\frac{1}{2^{\circ}} / \cos \phi_{m} \times \frac{1}{2^{\circ}}$ grid (a-d) and interpolated on a $\frac{1}{8^{\circ}} / \cos \phi_{\mathrm{m}} \times \frac{1}{8^{\circ}}$ grid (e-h) in km. Contour spacing is $50 \mathrm{~km}$. Only subregions with more than $50 \%$ wet grid points are shown.

[16] The horizontal resolution of the model allows testing the impact of different grid spacing in the subregions. Figure 4 compares the length scales $L_{\max }, L_{0}, L_{1}$, and $L_{*}$ (again with different scaling) for subregions with grid spacing of either $0.5^{\circ}$ or $0.125^{\circ}$, while Figures $3 \mathrm{e}-3 \mathrm{~h}$ show results with grid spacing of $0.25^{\circ}$. The maximum of $E(k)$, $k_{\max }$, and the first zero-crossing of $R(\tau), L_{0}$, are not much effected by the different grid spacing, while the integral length scale $L_{1}$ shows a rather strong impact. This effect is much less pronounced in $L_{*}$, showing only near the equator a markable impact by the different interpolations. This is because the integral $\int k^{2} P^{2}(k) \mathrm{d} k$ in equation (7) depends strongly on the higher wave numbers, while the highest power of $k$ in equation (8) is one which shows much less dependency on higher wave numbers. Note that the application of envelopes (or data windows like Hamming or triangular) to the data prior to the FFT affects the results in a similar way as the different grid spacing (not shown): While a)

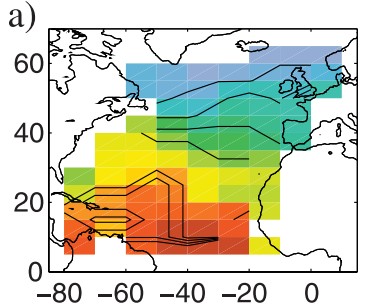

c)

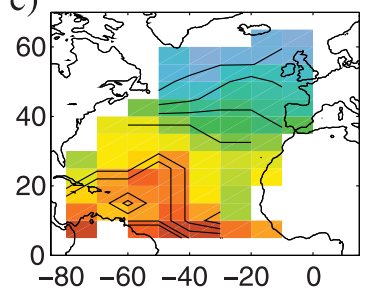

e)

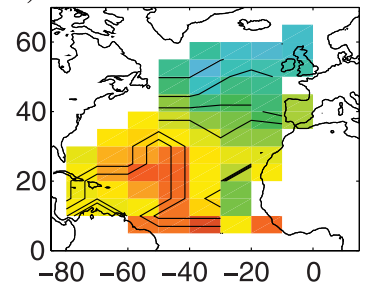

b)

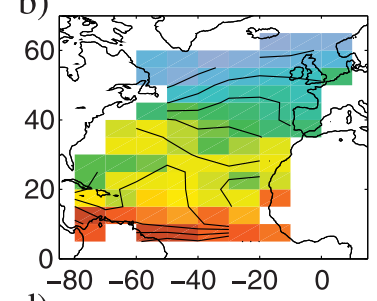

d)

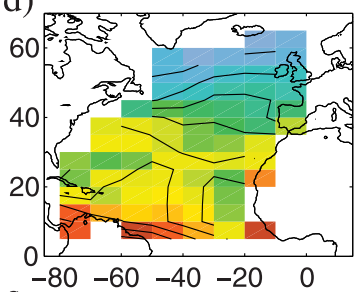

f)

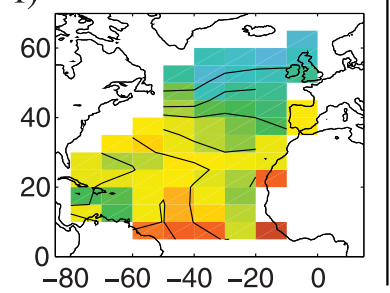

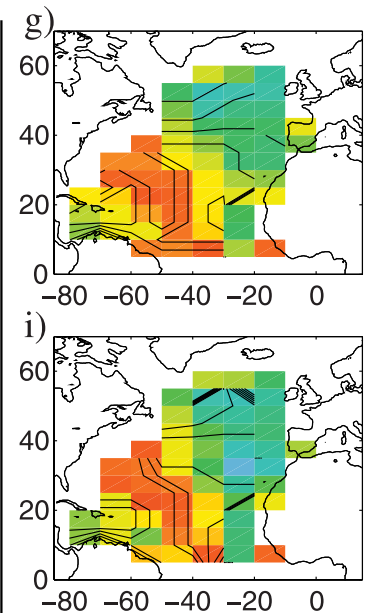

$\mathrm{k})$

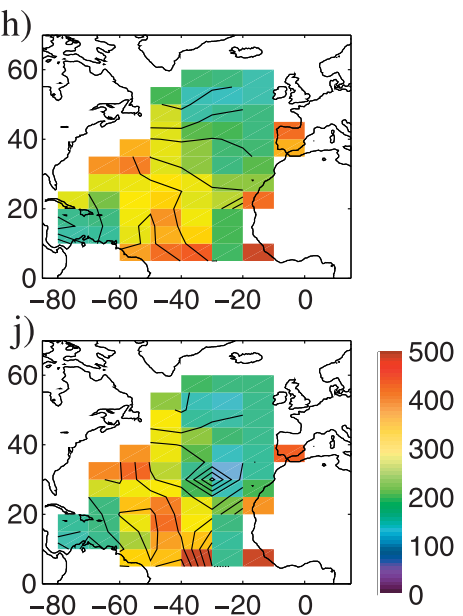

1)
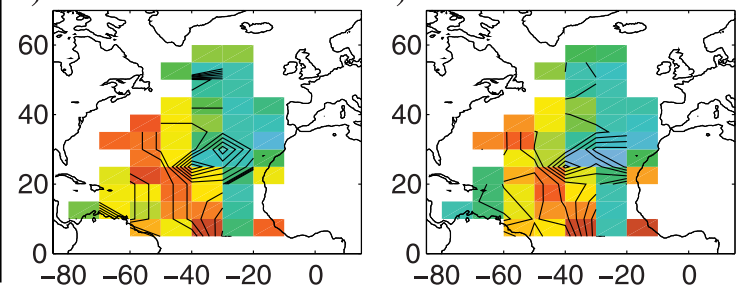

Figure 5. Length scale $0.5 L_{*}^{(x)}(\mathrm{a}, \mathrm{c}, \mathrm{e}, \mathrm{g}, \mathrm{i}, \mathrm{k})$ and $0.5 L_{*}^{(y)}(\mathrm{b}, \mathrm{d}, \mathrm{f}, \mathrm{h}, \mathrm{j}, \mathrm{l})$ estimated from surface pressure $(\mathrm{a}, \mathrm{b})$, pressure at $100 \mathrm{~m}(\mathrm{c}, \mathrm{d}), 500 \mathrm{~m}(\mathrm{e}, \mathrm{f}), 1000 \mathrm{~m}(\mathrm{~g}, \mathrm{~h}), 1500 \mathrm{~m} \mathrm{(i,j)}$, and $2000 \mathrm{~m} \mathrm{(k,} \mathrm{l)} \mathrm{in} \mathrm{km.} \mathrm{Only}$ subregions with more than $50 \%$ wet grid points at the respective depth are shown. 
a)

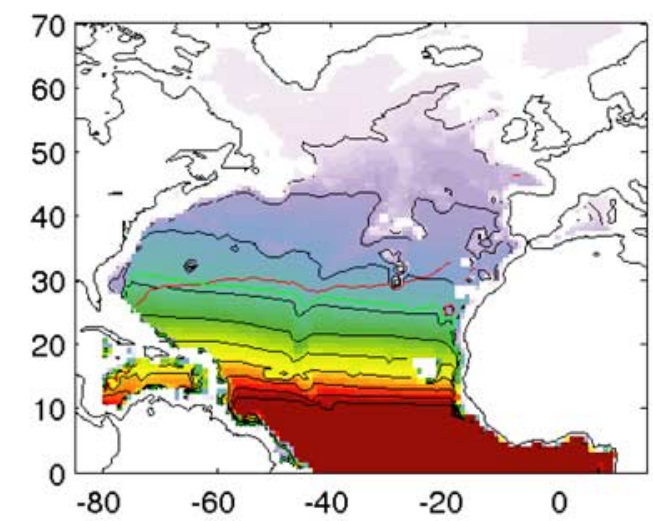

b)

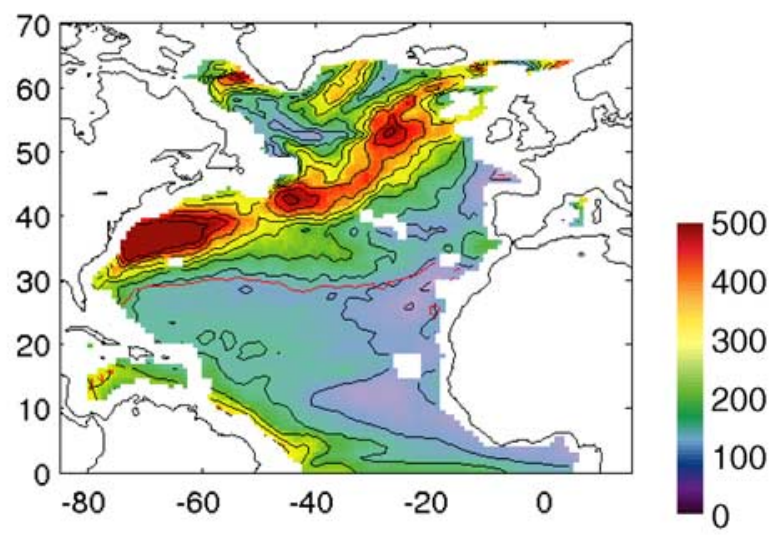

Figure 6. (a) Rossby radius $2 \pi L_{\mathrm{r}}$ in $\mathrm{km}$ and (b) Rhines scale $2 \pi L_{\mathrm{R}}$ in $\mathrm{km}$. Black lines denote same quantity as color shading with contour interval of $50 \mathrm{~km}$. The red line connects points at which $L_{\mathrm{r}}=L_{\mathrm{R}}$. Green line in Figure 6a denotes points at which $L_{\mathrm{r}}=2 \pi \times 30 \mathrm{~km}$.

$L_{\max }, L_{0}$, and $L_{*}$ show almost no difference using different data windows, the integral length scale $L_{1}$ shows similar, although smaller, differences as using higher (lower) resolution. This points again toward the dependency of $L_{1}$ on higher wave numbers, for which different data windowing methods appear to be more important. Note also that the size of different subregions might also influence the results shown here, although all estimated length scales are well below the size of the subregions.

[17] It is therefore concluded here that the classical integral length scale $L_{1}$ is for the present application less useful, and that the length scale $L_{*}$ resembles a better, i.e., more stable, integral scale both for the model results and the observations. Since model and observations agree well in all spectral properties of the surface pressure, the discussion in the remainder of this study will be devoted to the model results.

\subsection{Nonisotropy, Lateral, and Vertical Dependency of the Eddy Length Scale}

[18] Figure 5 shows the length scales $L_{*}^{(x)}$ and $L_{*}^{(y)}$ (again with the same scaling as for $L_{*}$ in Figure 3 ) at different depths over the thermocline of the North Atlantic Ocean. $L_{*}^{(x)}$ and $L_{*}^{(y)}$ tend to be of same magnitude north of about $30^{\circ} \mathrm{N}$, while $L_{*}^{(x)}$ is considerably larger than $L_{*}^{(y)}$ in the western subtropical North Atlantic. This anisotropy in the western subtropical North Atlantic can also be found at any depth range (Figure 5) and in the satellite observations as well (not shown). In general, eddy length scales do not vary much with depth, although north of the subpolar front, $L_{*}^{(x)}$ and $L_{*}^{(y)}$ tend to be larger at larger depths. Note that this depth dependency can also be seen in the length scales $L_{\max }$ and $L_{0}$ (not shown).

[19] The estimated length scales appear to differentiate two different regimes in the North Atlantic: The region north of about $30^{\circ} \mathrm{N}$ is characterized by isotropic turbulence, while south of this latitude, the turbulent flow tends to be anisotropic. In the anisotropic regime, the zonal fluctuations appear to be larger than the meridional ones, which is characteristic of two-dimensional turbulence on a $\beta$-plane [Rhines, 1975, 1977].

[20] The characteristic eddy length scales estimated from model simulations or observations are often compared with the first baroclinic Rossby radius [Krauss et al., 1990; a)

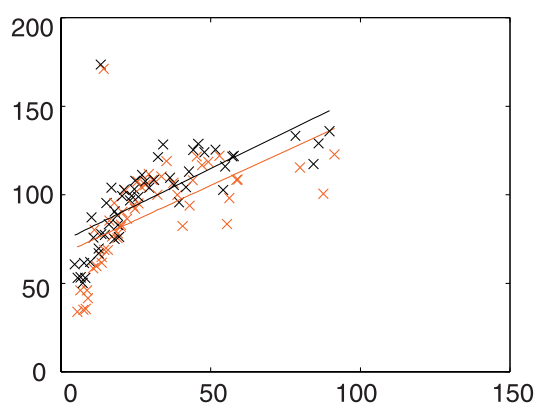

b)

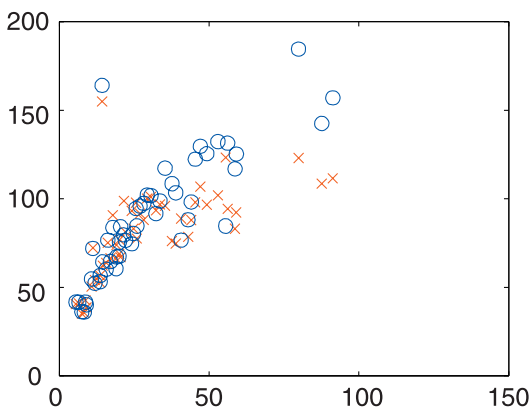

c)

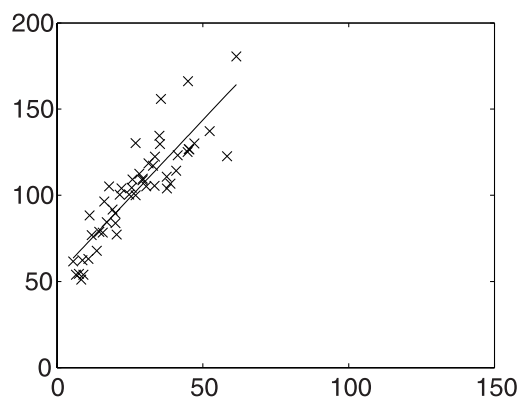

Figure 7. (a) Length scale $L_{0}$ at the surface versus first baroclinic Rossby radius $L_{\mathrm{r}}$ in km in JASON (black crosses) and model (red crosses). Solid lines denote regressions $L_{0}$ versus $L_{\mathrm{r}}$ for JASON (black, $L_{0}=0.8 L_{\mathrm{r}}+$ $74 \mathrm{~km}$ ) and the model (red, $L_{0}=0.8 L_{\mathrm{r}}+66 \mathrm{~km}$ ). (b) Length scales $1 / k_{*}^{(x)}$ (blue) and $1 / k_{*}^{(y)}(\mathrm{red})$ versus $L_{\mathrm{r}}$ in $\mathrm{km}$ in the model simulation. (c) $L_{0}$ in JASON versus the minimum of the local Rossby radius $L_{\mathrm{r}}$ and the local Rhines scale $L_{\mathrm{R}}$ in $\mathrm{km}$. 
Beckmann et al., 1994; Stammer and Böning, 1996]. The first baroclinic Rossby radius $L_{\mathrm{r}}$ is shown in Figure 6a. $L_{\mathrm{r}}$ is estimated approximately from the time mean model stratification following the work of Chelton et al. [1998] by calculating $L_{\mathrm{r}}=\int_{-H \mid}^{0} \frac{N}{|f| \pi} \mathrm{d} z$, where $N$ denotes the local buoyancy frequency and $f$ is the Coriolis parameter. The variations of $L_{\mathrm{r}}$ are to first order determined by the variation of $f$ and thus by latitude. Since all eddy length scales also vary with latitude, it is tempting to look for a relationship between $L_{\mathrm{r}}$ and the eddy lengths scales.

[21] By a regression, Stammer [1997] found a linear relationship between the Rossby radius $L_{\mathrm{r}}$ and $L_{0}$ estimated from satellite altimeter data. A very similar relation is found in the present study both in the model and observations. Figure 7a shows $L_{0}$ (at the surface) versus $L_{\mathrm{r}}$ for the model simulation and the satellite altimeter data in the North Atlantic from JASON. A linear fit using all data points gives in both cases a slope of 0.8 , very similar to the value given by Stammer [1997]. On the other hand, it is evident from Figure $7 \mathrm{a}$ that the relation between $L_{0}$ and $L_{\mathrm{r}}$ is far from linear. For small $L_{\mathrm{r}}$, the slope gets much steeper than 0.8 , and for $L_{\mathrm{r}}$ larger than about $30 \mathrm{~km}, L_{0}$ does not show much dependency on $L_{\mathrm{r}}$ anymore. Note that a similar behavior of $L_{0}$ versus $L_{\mathrm{r}}$ was found by Stammer [1997] (compare his Figure 24) for the global ocean. Note also that Krauss et al. [1990] found a slope of larger than 3 in a combined analysis of drifter data and satellite surface temperature observations which was restricted to the subpolar North Atlantic. If the linear regression in Figure $7 \mathrm{a}$ is repeated using data for $L_{\mathrm{r}}<30 \mathrm{~km}$ only (not shown), the estimated linear regressions are $L_{0}=2.25 L_{\mathrm{r}}+48 \mathrm{~km}$ for the JASON data and $L_{0}=3.15 L_{\mathrm{r}}+21.38 \mathrm{~km}$ for the model data. This means that restricting the regression to the subpolar North Atlantic, the slopes are much steeper than those reported by Stammer [1997] and consistent with the analysis by Krauss et al. [1990].

[22] Figure 7b shows the length scales $L_{*}^{(x)}$ and $L_{*}^{(y)}$ at the surface in the model also as a function of the local Rossby radius $L_{\mathrm{r}}$. For $L_{\mathrm{r}}$ smaller about $30 \mathrm{~km}$, both length scales show the same dependency on $L_{\mathrm{r}}$ (both with a slope of ca. 3) pointing toward isotropy. However, for $L_{\mathrm{r}}$ larger than about $30 \mathrm{~km}, L_{x}$ tends to be $20-100 \%$ larger than $L_{y}$ which is again characteristic of two-dimensional turbulence on a $\beta$-plane and which points again toward two different dynamical regimes of the turbulent flow in the North Atlantic.

[23] The characteristic length scale for two-dimensional turbulence on a $\beta$-plane is given by the Rhines scale [Rhines, $1975,1977]$. The Rhines scale $L_{\mathrm{R}}$ is shown in Figure $6 \mathrm{~b}$ and is calculated from the model results as $L_{\mathrm{R}}=\left(\frac{u_{\mathrm{rms}}}{2 \beta}\right)^{1 / 2}$ where $u_{\text {rms }}$ denotes a turbulent barotropic velocity scale taken here as $u_{\mathrm{rms}}^{2}=\left|\boldsymbol{u}_{\mathrm{b}}-\overline{\boldsymbol{u}}_{\mathrm{b}}\right|^{2}$ with $\boldsymbol{u}_{\mathrm{b}}=\left|h^{-1} \int_{-h}^{0} \boldsymbol{u d} z\right|$ and the temporal mean $\overline{\boldsymbol{u}}_{\mathrm{b}}$. Note that there is some ambiguity in the definition of the Rhines scale. Here the turbulent velocity scale $u_{\mathrm{rms}}$ is derived using the barotropic velocity, but one might also choose the (depth-averaged) first baroclinic mode velocity fluctuations or the local velocity fluctuations for the definition of $u_{\mathrm{rms}}$. However, it is shown in the study by C. Eden (Towards a turbulence model for meso-scale eddies, submitted to Journal of Physical Oceanography, 2007, hereinafter referred to as C. Eden, submitted manuscript, 2007) that for the present model, the ratio of the turbulent energy contained in the barotropic mode to the total turbulent energy (EKE) stays between 0.4 and 0.6 over most of the North Atlantic (only near the equator the ratio gets small). Thus the above choice for $L_{\mathrm{R}}$ is not much affected by using baroclinic or barotropic velocities for the definition of $u_{\mathrm{rms}}$. Although $L_{\mathrm{R}}$ depends on latitude via $\beta$, variations of $u_{\mathrm{rms}}$ dominate in $L_{\mathrm{R}}$. The Rhines scale $L_{\mathrm{R}}$ is larger than the Rossby radius $L_{\mathrm{r}}$ north of about $30^{\circ} \mathrm{N}$, which coincides roughly with the contour $L_{\mathrm{r}}=2 \pi \times 30 \mathrm{~km}$. Note that the latitude $30^{\circ} \mathrm{N}$ or the value $L_{\mathrm{r}}=2 \pi \times 30 \mathrm{~km}$ separates the anisotropic from the isotropic regions in Figures 5, 7a, and $7 \mathrm{~b}$.

[24] Figure 7c shows the scalar length scale $L_{0}$ in JASON versus the minimum of the local Rossby radius and the local Rhines scale from the model simulation. It is clear from the figure that to a reasonable approximation, a linear relationship shows up between the estimated length scales $L_{0}$ and $\min \left(L_{\mathrm{r}}, L_{\mathrm{R}}\right)$, much better as before using the Rossby radius only. A very similar result is obtained estimating the length scale in the model. It is concluded here that in the region of anisotropic (isotropic) turbulence, the length scale $L_{0}$ is linearly related to the Rhines scale $L_{\mathrm{R}}$ (Rossby radius $L_{\mathrm{r}}$ ), and that the scale given by $\min \left(L_{\mathrm{r}}, L_{\mathrm{R}}\right)$ is able to fit the estimated length scale $L_{0}$ well in both regions.

\section{Summary and Discussion}

[25] Eddy length scales are estimated in the North Atlantic Ocean both in satellite observations and a realistic highresolution model simulation. Remarkably good agreement between model and observations was found with respect to the analyzed spectral properties of surface pressure in horizontal wave number space. In the subpolar North Atlantic, however, eddy kinetic energy peaks at a slightly larger scale in the observations compared to the model. This artifact might be related to the smoothing in the satellite data involved during preparation of the gridded data product from the raw satellite tracks.

[26] A prominent feature of the kinetic energy spectrum $E(k)$, both in the model and observations, is that the peak of $E(k)$, at the wave number $k_{\max }$, moves to smaller length scales going northward. This latitudinal dependency consistently shows up as well in the first zero-crossing of the spatial autocorrelation function $L_{0}$ and in the integral length scale $1 / k_{*}$ (the latter was slightly redefined here from the classical definition for the present purpose). A characteristic eddy length scale can be defined now from the more heuristic length scales $L_{\max }$ or $L_{0}$ as proposed by, e.g., Stammer [1997] or, maybe more theoretically sound, from the integral length scale $L_{*}$, in order to estimate lateral diffusivities in the North Atlantic (given a characteristic velocity scale as well).

[27] The integral eddy length scale was formulated here also as a vector length scale to detect a possible anisotropy of the turbulent flow. It is found that in the western subtropical North Atlantic, the zonal integral length scales are $20-100 \%$ larger compared to the meridional integral length scales, indicative of anisotropic turbulent flow, while north of about $30^{\circ} \mathrm{N}$, the magnitudes of both length scales are very similar (isotropic turbulence). The length scale of turbulent geostrophically balanced flow as a function of depth is also discussed in the present paper. Anisotropic versus isotropic regions and eddy length scales in the sub- 
tropical North Atlantic change hardly with depth pointing toward only weak depth dependency of the spectral properties of the turbulent flow, although the eddy kinetic energy decays with depth (not shown, but see, e.g., Smith et al. [2000]). On the other hand, the (small) length scales in the subpolar North Atlantic tend to increase slightly with depth. The reason for this feature remains unclear, but a possible explanation for this feature might be that the length scales for barotropic and baroclinic modes are different and that the vertical localization of barotropic and baroclinic kinetic energies with depth depends on latitude. However, a full assessment of modal energies, energy fluxes, and length scales in the model is beyond the scope of the present study.

[28] The question of a possible relation between the first Rossby radius and the characteristic eddy length scale is revisited here. It was argued previously by Le Traon et al. [1990] and Stammer and Böning [1992] that the latitudinal variation of the length scale might be indicative of a linear relation with the first baroclinic Rossby radius, although it was also noted by Le Traon [1993] that while the Rossby radius varies by a factor of 4 , the length scales vary only by a factor of 2. This feature is also prominent from the apparent nonlinearity when plotting the estimated eddy length scales as a function of the local Rossby radius (compare Figure 7). On the other hand, Krauss et al. [1990] reported a closer linear relation between Rossby radius and eddy length scale in an analysis based on surface drifter data and infrared satellite images which was restricted to the northern North Atlantic.

[29] It is shown here that a close linear relation can only be found in the North Atlantic for Rossby radii smaller than about $30 \mathrm{~km}$ or north of about $30^{\circ} \mathrm{N}$, i.e., for the region of isotropic turbulent flow. The estimated slope between eddy length scale and the Rossby radius for the subpolar North Atlantic is similar to the one found by Krauss et al. [1990], i.e., much steeper than the slope given by, e.g., Stammer [1997] from a regression including subtropical and subpolar regions. On the other hand, the eddy length scales show no clear dependency on the Rossby radius south of $30^{\circ} \mathrm{N}$ or for Rossby radii larger than about $30 \mathrm{~km}$. Here the turbulent flow becomes anisotropic, with zonal length scales larger than meridional ones, indicative of two-dimensional turbulence on a $\beta$-plane [Rhines, 1975, 1977].

[30] The dominant length scale for such a flow regime is given by the Rhines scales $L_{\mathrm{R}}$. It is important to note that in the model, $L_{\mathrm{R}}$ becomes smaller than the local Rossby radius for Rossby radii smaller than $30 \mathrm{~km}$ or south of $30^{\circ} \mathrm{N}$. It is argued here that for meridional fluctuations, i.e., for the meridional eddy length scale, in the region south of $30^{\circ} \mathrm{N}$, $L_{\mathrm{R}}$ might be the more relevant length scale. Because of such a limitation of the meridional length scale by $L_{R}$, the estimated (scalar) eddy length scale in the altimeter data and the model simulation show no clear dependence on the local Rossby radius $L_{\mathrm{r}}$ in the subtropical North Atlantic. Instead, it was found here that the estimated eddy length scale is linearly related to the local Rhines scale in that region. As a consequence, the length scale given by $\min \left(L_{\mathrm{r}}\right.$, $\left.L_{\mathrm{R}}\right)$ is able to fit the scalar eddy length scale reasonably well over the entire basin.

[31] Support of this interpretation comes from a recent study by Theiss [2004]. In an idealized, eddying shallow water model including the latitudinal variation of the
Rossby radius (i.e., considering not just a $\beta$-plane with fixed Rossby radius as in previous studies), he found a critical latitude, above which the flow is isotropic and below which the flow becomes anisotropic (zonal scales larger than meridional ones). This critical latitude is defined by $L_{\mathrm{r}}=L_{\mathrm{R}}$. Where the Rhines scale $\left(L_{\mathrm{R}}\right)$ is larger than the local Rossby radius $\left(L_{\mathrm{r}}\right)$, i.e., north of the critical latitude, the flow is isotropic; when the Rhines scale becomes smaller than the Rossby radius, the flow becomes anisotropic. This result by Theiss [2004] is also confirmed by a recent study by R. K. Scott and L. M. Polvani (Forced dissipative shallow water turbulence on the sphere: equatorial confinement of zonal jets, submitted to Journal of the Atmospheric Sciences, 2007, hereinafter referred to as R. K. Scott and L. M. Polvani, submitted manuscript, 2007). Note that the prediction of such a critical latitude based on the ratio of Rossby radius and Rhines scale is surprisingly consistent with the present study. However, note also that the idealized studies by Theiss [2004] and R. K. Scott and L. M. Polvani (submitted manuscript, 2007) deal with decaying turbulence, in which eddy length scales might be different from the more realistic forced/dissipated case as considered in the present study.

[32] A similar difference in the turbulent flow regime (zonally elongated near the equator and isotropic near the poles) can be found from images of Jupiter's atmosphere, serving usually as a popular example for geostrophic turbulence [Theiss, 2006]. Further support comes from the recent evidence from observational and modeling studies concerning strong zonal jets in the equatorial to subtropical Atlantic and Pacific Ocean [Schott et al., 2003; Maximenko et al., 2005; Eden, 2006b], also indicative of geostrophic turbulence on a $\beta$-plane and anisotropic flow in the tropical and subtropical ocean.

[33] Finally, it should be noted that in the study by C. Eden (submitted manuscript, 2007), it was attempted to construct a lateral diffusivity (in the interior of the ocean essentially given by the thickness diffusivity in the work of Gent and McWilliams [1990]) following the ideas by Green [1970], Stone [1972], and Larichev and Held [1995], i.e., using a characteristic length and velocity scale (where the latter is given by a prognostic eddy kinetic energy budget). Such a diffusivity yields only results consistent with independent estimates given by Eden et al. [2006], defining the eddy length scale as the minimum between (essentially) the local Rossby radius and Rhines scale, consistent with the results of the present study. It should be stressed that using a previous suggestion by Visbeck et al. [1997], i.e., using a length scale based on the Rossby radius only, the resulting diffusivity is in disagreement with the independent estimates.

[34] Acknowledgments. The model integrations have been performed on a NEC-SX8 at the computing center at the University Kiel, Germany, and on a NEC-SX6 at the Deutsches Klimarechenzentrum (DKRZ), Hamburg, Germany. Two anonymous reviewers helped to improve the manuscript.

\section{References}

Barnier, B., L. Siefridt, and P. Marchesiello (1995), Thermal forcing for a global ocean circulation model using a three year climatology of ECMWF analysis, J. Mar. Syst., 6, 363-380.

Batchelor, G. K. (1969), Computation of the energy spectrum in homogeneous two-dimensional turbulence, Phys. Fluids Suppl., 12B, 233 239 . 
Beckmann, A., C. W. Böning, C. Köberle, and J. Willebrand (1994), Effects of increased horizontal resolution in a simulation of the North Atlantic Ocean, J. Phys. Oceanogr., 24, 326-344.

Bryan, K., J. K. Dukowicz, and R. D. Smith (1999), On the mixing coefficient in the parameterization of bolus velocity, J. Phys. Oceanogr., 29, $2442-2456$.

Chelton, D. B., R. A. deSzoeke, M. G. Schlax, K. E. Naggar, and N. Siwertz (1998), Geographical variability of the first-baroclinic Rossby radius of deformation, J. Phys. Oceanogr., 28, 433-460.

Eden, C. (2006a), Mid-depth equatorial tracer tongues in a model of the Atlantic Ocean, J. Geophys. Res., 111, C12025, doi:10.1029/2006JG003565.

Eden, C. (2006b), Thickness diffusivity in the Antarctic Circumpolar Current, Geophys. Res. Lett., 33, L11606, doi:10.1029/2006GL026157.

Eden, C., and C. W. Böning (2002), Sources of eddy kinetic energy in the Labrador Sea, J. Phys. Oceanogr., 32(12), 3346-3363.

Eden, C., R. J. Greatbatch, and J. Willebrand (2006), A diagnosis of thickness fluxes in an eddy-resolving model, J. Phys. Oceanogr., in press.

Gent, P. R., and J. C. McWilliams (1990), Isopycnal mixing in ocean circulation models, J. Phys. Oceanogr., 20, 150-155.

Green, J. S. (1970), Transfer properties of the large-scale eddies and the general circulation of the atmosphere, Q. J. R. Meteorol. Soc., 96, $157-185$.

Houghton, J. T., Y. Ding, D. J. Griggs, M. Noguer, P. J. van der Lindeni, X. Dai, K. Maskell, and C. A. Johnson (Eds) (2001), in Climate Change (2001) The scientific basis, chapter Contribution of Working Group 1 to the Third Assessment Report of the Intergovernmental Panel on Climate Change, p. 881, Cambridge Univ. Press, New York.

Krauss, W., R. Döscher, A. Lehmann, and T. Viehoff (1990), On eddy scales in the eastern and northern North Atlantic Ocean as a function of latitude, J. Geophys. Res., 95(C10), 18,049-18,056.

Larichev, V., and I. Held (1995), Eddy amplitudes and fluxes in a homogenous model of fully developed baroclinic instability, J. Phys. Oceanogr. 25, 2285-2297.

Le Traon, P. Y. (1993), Comments on mesoscale variability in the Atlantic Ocean from Geosat altimetry and WOCE high-resolution numerical modeling, J. Phys. Oceanogr., 23, 2729-2732.

Le Traon, P. Y., M. C. Rouquet, and C. Boissier (1990), Spatial scales of mesoscale variability in the North Atlantic as deduced from Geosat data, J. Geophys. Res., 95, 20,267-20,285.

Maximenko, N. A., B. Bang, and H. Sasaki (2005), Observational evidence of alternating zonal jets in the world ocean, Geophys. Res. Lett., 32, L12607, doi:10.1029/2005GL022728.

Pacanowski, R. C. (1995), MOM 2 Documentation, User's Guide and Reference Manual, Technical report, GFDL Ocean Group, GFDL, Princeton, N. J., USA.
Prandtl, L. (1925), Bericht über Untersuchungen zur ausgebildeten Turbulenz, Z. Angew. Math. Mech., 5(2), 136-139.

Rhines, P. (1975), Waves and turbulence on a beta-plane, J. Fluid Mech., 69, 417-443.

Rhines, P. (1977), The Sea, volume 6, chapter The dynamics of unsteady currents, pp. 189-318, John Wiley, Hoboken, N. J.

Schott, F., M. Dengler, P. Brandt, K. Affler, J. Fischer, B. Bourles, Y. Gouriou, R. Molinari, and M. Rhein (2003), The zonal transports at $35^{\circ} \mathrm{W}$ in the tropical Atlantic, Geophys. Res. Lett., 30(7), 1349, doi:10.1029/ 2002GL016849.

Smith, R. D., M. E. Maltrud, F. O. Bryan, and M. W. Hecht (2000), Numerical simulation of the North Atlantic Ocean at $1 / 10^{\circ}$, J. Phys. Oceanogr., 30, 1532-1561.

Stammer, D. (1997), Global characteristics of ocean variability estimated from regional TOPEX/POSEIDON altimeter measurements, J. Phys. Oceanogr., 27, 1743-1769.

Stammer, D., and C. W. Böning (1992), Mesoscale variability in the Atlantic Ocean from Geosat altimetry and WOCE high-resolution numerical modeling, J. Phys. Oceanogr., 22, 732-752.

Stammer, D., and C. W. Böning (1996), The Warmwatersphere of the North Atlantic Ocean, chapter Generation and distribution of mesoscale eddies in the North Atlantic Ocean, Gebrüder Bornträger, Berlin, Germany.

Stevens, D. P. (1990), On open boundary conditions for three dimensional primitive equation ocean circulation models, Geophys. Astrophys. Fluid Dyn., 51, 103-133.

Stone, P. H. (1972), A simplified radiative-dynamical model for the static stability of rotating atmospheres, J. Atmos. Sci., 29, 405-418.

Theiss, J. (2004), Equatorward energy cascade, critical latitude, and the predominance of cyclonic vortices in geostrophic turbulence, J. Phys. Oceanogr., 34, 1663-1678.

Theiss, J. (2006), A generalized Rhines effect and storms on Jupiter, Geophys. Res. Lett., 33(L08809), doi:10.1029/2005GL025379.

Thompson, A., and W. Young (2006), Scaling baroclinic eddy fluxes: Vorticities and energy balance, J. Phys. Oceanogr., 36, 720-738.

Visbeck, M., J. Marshall, T. Haine, and M. Spall (1997), Specification of eddy transfer coefficients in coarse-resolution ocean circulation models, J. Phys. Oceanogr., 27, 381-402.

C. Eden, FB I Ocean Circulation and Climate Dynamics, IFM-GEOMAR, Düsternbrooker Weg 20, 24105 Kiel, Germany. (ceden@ifm-geomar.de) 